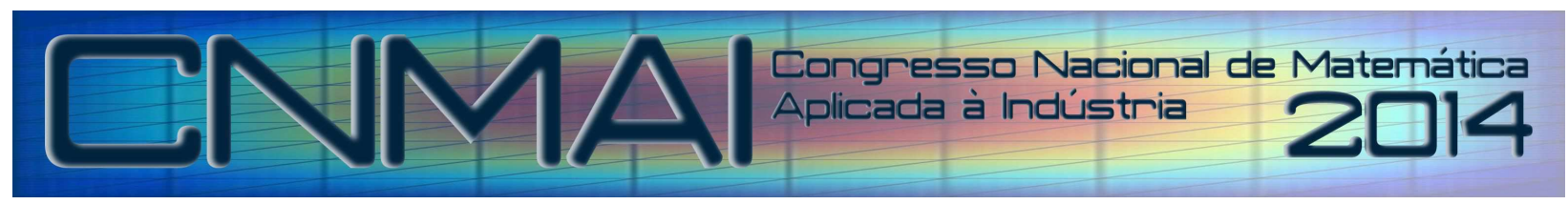

18 a 21 de novembro de 2014, Caldas Novas - Goiás

\title{
ESTUDOS DE SINGULARIDADES NO FLUXO DE CARGA CONTINUADO
}

\author{
Alfredo Bonini Neto ${ }^{1}$, e-mail: bonini@tupa.unesp.br \\ Elisabete de Mello Magalhães ${ }^{2}$, e-mail: elisabete.magalhaes@yahoo.com.br \\ Dilson Amancio Alves, e-mail: dalves@dee.feis.unesp.br \\ ${ }^{1}$ Universidade Estadual Paulista - UNESP - Campus de Tupã - Departamento de Engenharia de Biossistemas. Av. \\ Domingos da Costa Lopes, 780 - CEP 17602-496 \\ ${ }^{2}$ Universidade Estadual Paulista - UNESP - Campus de Ilha Solteira - Departamento de Engenharia Elétrica. Av. \\ Brasil, 56 - CEP 15385-000
}

\begin{abstract}
Resumo: Nos últimos anos a preocupação com o assunto estabilidade de tensão vem ganhando destaque mundial quando se refere à indústria do setor energético, isto se deve ao fato desta questão está relacionada com a operação e planejamento de sistemas elétricos de potência. Fatores como o crescente aumento da demanda de energia, a transferência de elevadas quantidades de potência para atender o consumo, aliados as exigências econômicas e ambientais têm levado os sistemas a operarem em condições estressantes (próximos de seus limites), ou seja, com pequenas margens de segurança que é uma ameaça à sua condição de operação estável. A combinação desses fatores pode ser desastrosa, pois tornam os sistemas elétricos de potência vulneráveis, ou seja, expostos ao risco de uma situação de instabilidade. Na literatura, um dos estudos para análise de estabilidade e instabilidade de tensão está relacionado à curva $P-V$ (potência versus magnitude da tensão) e o ponto na curva que separa a operação estável da instável denomina-se como ponto de máximo carregamento. O ponto de máximo carregamento pode ser consequente de uma bifurcação de sela-nó (BSN) relacionada ao limite de capacidade de transmissão num sistema elétrico, onde a matriz Jacobiana é singular, ou de uma bifurcação estática induzida por limites (BIL), relacionada com o limite de potência reativa do gerador, onde a matriz não é singular. Neste sentido, apresenta-se neste trabalho uma proposta para a melhoria do desempenho do fluxo de carga continuado visando à obtenção do ponto de máximo carregamento de um sistema elétrico de potência sem problemas numéricos relacionados à singularidade da matriz Jacobiana. Um estudo no ponto de máximo carregamento é efetuado com o intuito de verificar se o ponto corresponde a uma bifurcação sela-nó (BSN) ou a uma bifurcação estática induzida por limites (BIL).
\end{abstract}

Palavras-chave: Estabilidade de tensão, Ponto de máximo carregamento, Bifurcação, Fluxo de carga continuado.

\section{INTRODUÇÃO}

A análise de estabilidade estática de tensão vem, ao longo do tempo, ganhando um grande destaque nacional e internacional dentre os diversos estudos relacionados com o planejamento e a operação de sistemas elétricos. Este interesse é uma consequência direta do crescimento contínuo da demanda que, associado às restrições econômicas e ambientais, e à desregulamentação do setor elétrico, tem levado os sistemas elétricos a operar próximo de seus limites operacionais. Estas análises podem ser realizadas através da obtenção do perfil de tensão das barras em função de seu carregamento (curvas P-V). Estas curvas possibilitam a compreensão das condições de operação dos sistemas para diferentes carregamentos, e têm sido recomendadas pelas empresas do setor elétrico internacional (WSCC, 1998) e nacional (FTCT, 1999), para avaliação da estabilidade de tensão.

Um dos principais objetivos da análise estática de estabilidade de tensão é a obtenção do ponto de máximo carregamento (PMC) do sistema. Sua obtenção é importante tanto para o cálculo de margens de estabilidade quanto para a realização da análise modal, uma vez que é neste ponto que ela fornece informações para a determinação de medidas efetivas para o reforço do sistema, já que o PMC define a fronteira entre as regiões de operação estável e instável.

A obtenção do PMC tem sido realizada por meio de sucessivas soluções do fluxo de carga (FC) através da variação manual do carregamento do sistema. Este procedimento é realizado até que o processo iterativo deixe de convergir. Para fins práticos, este ponto é considerado como sendo o PMC. Entretanto, sabe-se que os problemas de convergência 
encontrados pelo FC convencional para a obtenção do PMC são consequentes das dificuldades numéricas associadas à singularidade da matriz Jacobiana (J). Os métodos de fluxo de carga continuado (FCC) superam as dificuldades numéricas acima mencionadas pela adição de equações parametrizadas (Alves et all., 2000), (Ajjarapu e Christy, 1992), (Chiang et all., 1995), (Seydel, 1994), (Yang et all, 2012), (Pama e Radman, 2009), (Li e Chiang, 2008) e (Bonini Neto e Alves, 2010), com isso o traçado completo do perfil de tensão é efetuado variando automaticamente o valor de um determinado parâmetro do sistema, sem preocupação com as singularidades das equações do fluxo de carga. A diferença entre os métodos de FCC está no modo como o novo parâmetro é escolhido e em como a singularidade é eliminada. As técnicas de parametrização mais utilizadas pelos FCC para eliminar a singularidade de $\mathbf{J}$ são a local (Ajjarapu e Christy, 1992) e a geométrica (Chiang et all., 1995). Mais recentemente, técnicas globais de parametrização também são utilizadas para contornar os problemas relacionados à singularidade da matriz J (Bonini Neto e Alves, 2010). A singularidade da matriz J é verificada através da inversão do sinal do determinante no PMC, ou seja, o determinante é nulo exatamente no PMC, podendo ser verificado em sistemas de pequeno porte por meio do gráfico dos valores numéricos do determinante em função do carregamento $\lambda$. A dimensão da matriz quadrada $\mathbf{J}$ é da ordem de $\left(2 n_{P Q}+n_{p v}\right)$, onde $n_{P Q}$ e $n_{p v}$ correspondem ao número de barras PQ e PV, respectivamente. Então, para um sistema como o IEEE-14 barras, que pode ser visto na Fig. 1, a matriz $\mathbf{J}$ convencional é da ordem de $23 \times 23(2 \times 9+5)$.

Neste trabalho é apresentada uma análise das singularidades da matriz $\mathbf{J}$ para dois sistemas, o IEEE-14 barras e outro de maior porte que é o sistema IEEE-300 barras. O PMC pode ser consequente ou de uma bifurcação sela-nó (BSN), relacionada ao limite de capacidade de transmissão de um sistema elétrico, ou de uma bifurcação estática induzida por limites (BIL) a qual está relacionada com o limite de potência reativa do gerador. Uma simples diferenciação entre as duas pode ser efetuada pela análise do determinante da matriz $\mathbf{J}$, a qual é singular no caso da BSN e não singular no caso da BIL. Entretanto, uma vez que para as diversas condições de carregamento analisadas os valores numéricos do determinante do sistema IEEE-300 são muito elevados, maiores do que 1 e+309, a sua representação no software MATLAB é efetuada pelo símbolo inf ( $\infty$, infinito), impossibilitando assim a sua visualização gráfica. Uma alternativa para viabilizar a representação gráfica dos valores de seu determinante em função do carregamento $\lambda$ é a redução dos seus valores através da divisão dos elementos da matriz por 10. Neste sentido, apresenta-se neste trabalho uma proposta para a melhoria do desempenho do fluxo de carga continuado visando à obtenção do ponto de máximo carregamento de um sistema elétrico de potência sem problemas numéricos relacionados à singularidade da matriz J. Um estudo no PMC é efetuado com o intuito de verificar se o ponto corresponde a uma bifurcação sela-nó (BSN) ou a uma bifurcação estática induzida por limites (BIL).

\section{METODOLOGIA}

Os métodos de FCC permitem o traçado completo das curvas $\mathrm{P}-\mathrm{V}$, a partir de um caso base, passando pelo PMC, por meio de técnicas de parametrizações. Em geral as equações de FC podem ser escritas da forma que se segue:

$$
\mathbf{G}(\mathbf{V}, \boldsymbol{\theta}, \lambda)=0
$$

em que:

V é o vetor das magnitudes de tensões nodais.

$\boldsymbol{\theta}$ é o vetor dos ângulos de fase nodais, excetuando o da barra de referência.

$\lambda$ é o fator de carregamento.

$\mathbf{G}$ é um vetor composto pelas equações dos balanços de potências ativa e reativa nodais.

Para este trabalho foram utilizadas diversas técnicas de parametrização para obtenção da curva P-V, parametrizado por $\lambda$, parametrizado pela magnitude de tensão da barra crítica ou de uma barra específica, (Ajjarapu e Christy, 1992), parametrizado por $\mathrm{Pa}$ (perdas totais de potência ativa) (Alves et all., 2000) e parametrizado por uma equação quadrática situada no plano $\lambda-P a$, ou seja, parábolas (Magalhães et all., 2013).

Para a técnica parametrizada por $\lambda$ a matriz $\mathbf{J}$ é dada por: (Ajjarapu e Christy, 1992)

$$
\left[\begin{array}{ccc}
\mathbf{G}_{\boldsymbol{\theta}} & \mathbf{G}_{\mathbf{V}} & \mathbf{G}_{\lambda} \\
\mathbf{0} & \mathbf{0} & 1
\end{array}\right]
$$

caso a magnitude de tensão de uma barra k seja escolhida como parâmetro do método da continuação, a matriz torna-se:

$$
\left[\begin{array}{ccc}
\mathbf{G}_{\boldsymbol{\theta}} & \mathbf{G}_{\mathbf{v}} & \mathbf{G}_{\lambda} \\
\mathbf{0} & {\left[0,0, \ldots, 1_{\mathrm{k}}, \ldots, 0\right]} & 0
\end{array}\right]
$$

se a técnica utilizada para o traçado da curva P-V for parametrizado por $\mathrm{Pa}$, a matriz $\mathbf{J}$ passa a ser: 


$$
\left[\begin{array}{ccc}
\mathbf{G}_{\boldsymbol{\theta}} & \mathbf{G}_{\mathbf{v}} & \mathbf{G}_{\lambda} \\
\partial P a / \partial \boldsymbol{\theta} & \partial P a / \partial \mathbf{V} & 0
\end{array}\right]
$$

e se a parametrização for dada pela equação quadrática (parábolas):

$$
\left[\begin{array}{ccc}
\mathbf{G}_{\boldsymbol{\theta}} & \mathbf{G}_{\mathbf{V}} & \mathbf{G}_{\lambda} \\
\partial P a / \partial \boldsymbol{\theta} & \partial P a / \partial \mathbf{V} & -(2 a(\lambda+\alpha)+b)
\end{array}\right]
$$

onde $a$ e $b$ são os coeficientes da função quadrática e $\alpha$ é a variável adotada como parâmetro e que determina a novas equações das parábolas (Magalhães et all., 2013).

\section{RESULTADOS}

Os estudos de singularidade da matriz $\mathbf{J}$ foram desenvolvidos para os sistemas do IEEE-14 e 300 barras. A Fig. 1 mostra o diagrama unifilar do sistema IEEE-14, representado por 5 barras de geração, 9 barras de carga e 20 linhas de transmissão (LT).

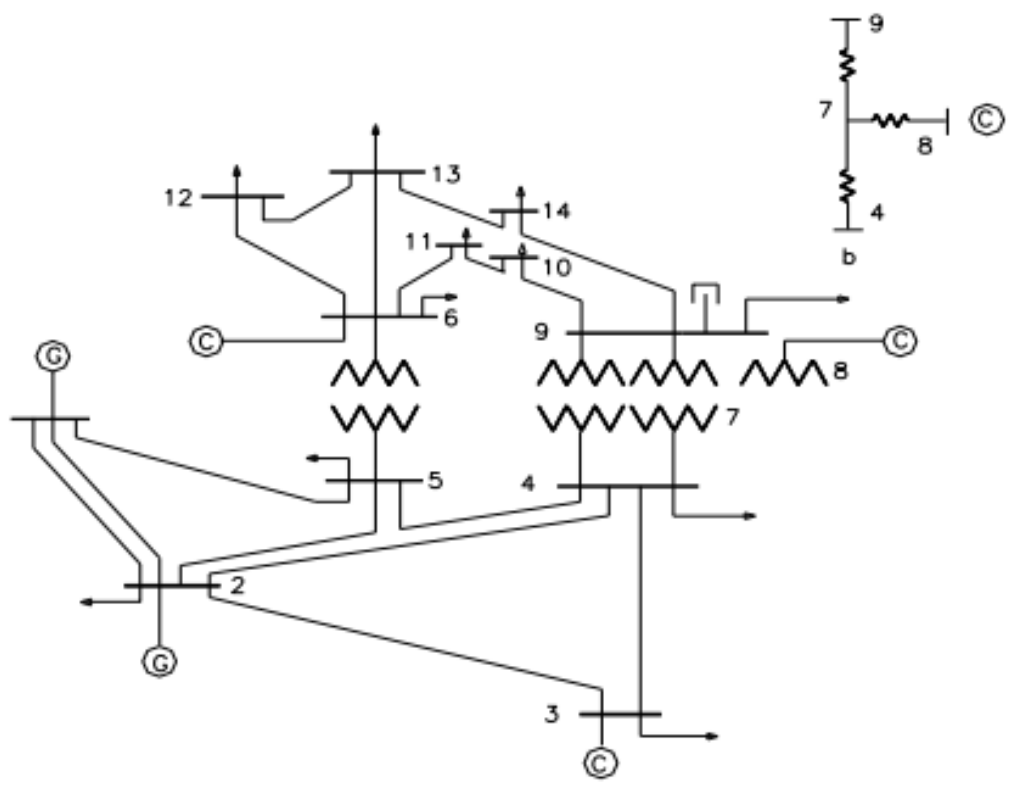

Figura 1: Diagrama unifilar do sistema IEEE-14.

A Fig. 2 mostra geometricamente a trajetória de soluções e os pontos de singularidade das respectivas matrizes Jacobianas para cada técnica utilizada neste trabalho. Observa-se que o único método que não apresenta problemas numéricos relacionados com a singularidade na obtenção da curva $\mathrm{P}-\mathrm{V}$ é o que usa a técnica de parametrização por parábolas, ver Fig. 2(a). Quando da utilização de $\lambda$ como parâmetro, o método fica restrito pelo ponto $S_{1}$, enquanto que parametrizado por $\mathrm{Pa}$ fica restrito pelo ponto $\mathrm{S}_{3}$, Fig. 2(b).
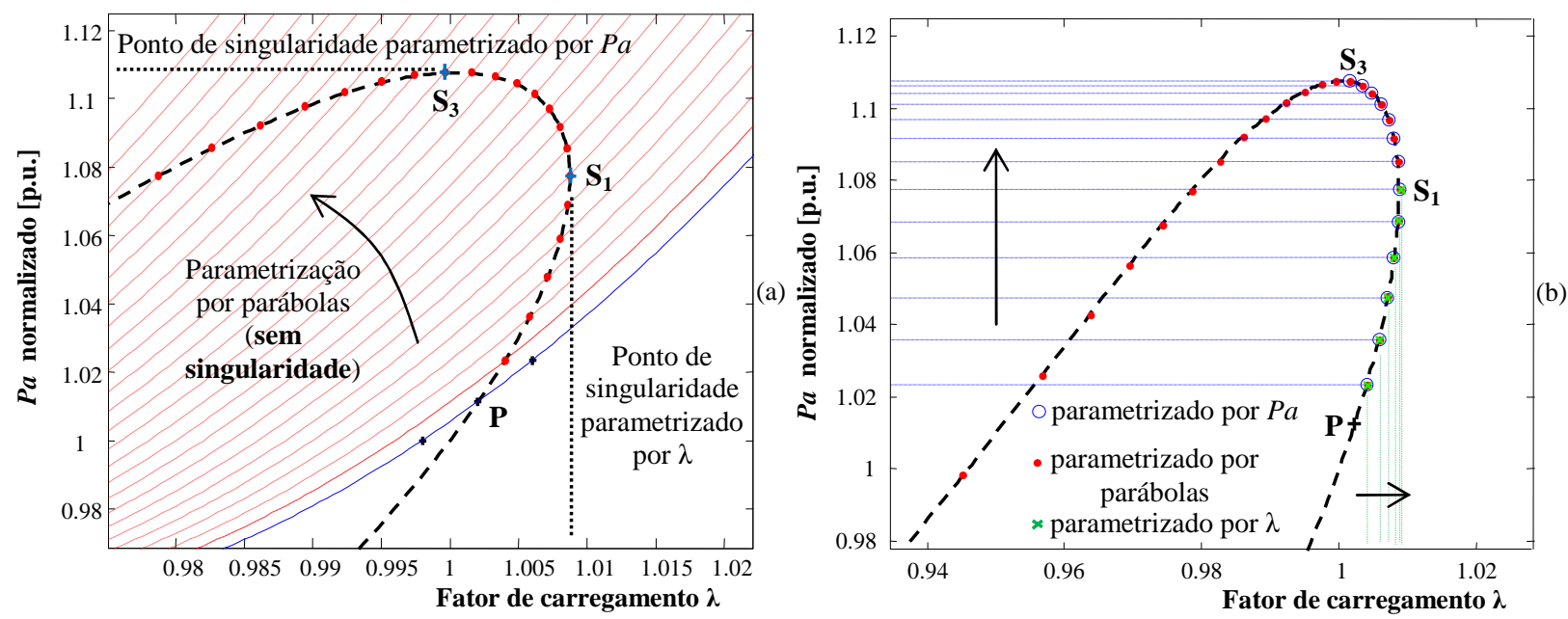

Figura 2: Geometria das soluções dos métodos estudados e respectivos pontos de singularidades na curva $\lambda$-Pa. 
Para o sistema IEEE-14 barras, os pontos de singularidade das matrizes Jacobianas e os respectivos valores dos determinantes para cada método é apresentado na Fig. 3. Na Fig. 3(a) são apresentadas as curvas P-V da barra de geração $2\left(\mathrm{~V}_{2}\right)$ e da crítica $\left(\mathrm{V}_{14}\right)$. Na Fig. 3(b) apresenta-se a curva perdas totais de potência ativa $(P a)$ em função de $\lambda$. Cada ponto de singularidade pode ser observado no gráfico dos valores dos determinantes das matrizes Jacobianas em função de $\lambda$, Fig. 3(c) e (d). Nota-se que se $\lambda$ for utilizado como parâmetro da continuação, o método falha na obtenção de pontos da curva $\mathrm{P}-\mathrm{V}$ que se encontram além do PMC, ou seja, fica como visto anteriormente limitado pelo ponto $S_{1}$ apresentado nas Figs. 3(a) e (b), isto é comprovado pelo valor do determinante da matriz $\mathbf{J}$, o qual se anula no ponto $S_{1}$, ver Fig. 3(c) e (d). No caso dos parâmetros magnitude de tensão da barra $2\left(\mathrm{~V}_{2}\right)$ e $P a$, constata-se que as matrizes Jacobianas são singulares nos respectivos pontos $S_{2}$ e $S_{3}$, e como é de se esperar os seus determinantes se anulam nesses pontos conforme se constatam na Fig. 3(d). Por outro lado, se a magnitude da tensão da barra crítica $\left(\mathrm{V}_{14}\right)$ ou a variável $\alpha$ da equação da parábola localizada no plano $\lambda-P a$ for utilizado como parâmetro, as suas matrizes não apresentam singularidades visto que os seus determinantes não intersectam o eixo da abscissa em nenhum ponto.

Para o sistema IEEE-14 barras, pode se observar que os gráficos dos determinantes se mantiveram contínuos ao longo de todo o traçado da curva P-V, comprovando que o PMC é consequente de uma bifurcação sela-nó (BSN) relacionada ao limite de capacidade de transmissão de um sistema elétrico, onde a matriz Jacobiana é singular.

A Fig. 4 apresenta os resultados para o sistema teste de 300 barras do IEEE. O sistema apresenta 231 barras de carga (PQ) e 68 barras de geração (PV), no entanto a matriz Jacobiana para este sistema é da ordem $530 \times 530$ acarretando em valores muito altos para o determinante. Nas figuras são mostradas duas curvas em função da mudança do tipo da barra de geração $\left(\mathrm{V}_{7062}\right)$ de $\mathrm{PV}$ para $\mathrm{PQ}$, muito próximo ao PMC. Essa mudança ocorre em função do limite máximo de geração de potência reativa $\left(\mathrm{Qg}_{\max }=1,5\right.$ p.u. de $\left.\mathrm{MVAr}\right)$ ter sido atingido. As curvas 1 e 2 são utilizadas para se avaliar se o PMC é causado por uma BSN ou uma BIL. A curva 1 é obtida utilizando-se os dados originais do banco de dados do sistema, enquanto que a curva 2 é traçada considerando-se a barra de geração 7062 (tipo PV originalmente) como sendo uma barra de carga (tipo PQ) com a sua potência reativa gerada fixa e igual ao seu valor máximo $\left(\mathrm{Q}_{\mathrm{g} 7062}=1,5\right.$ p.u. $\left.=\mathrm{Qg}_{\max (7062)}\right)$.

As correspondentes curvas P-V (curva 1 e 2 ) da barra crítica $\left(V_{526}\right)$ são apresentadas na Fig. 4(a), enquanto que as da barra $V_{7062}$ na Fig. 4(b). Na Fig. 4(c) são apresentadas as correspondentes curvas 1 e 2 das perdas totais de potência ativa $(\mathrm{Pa})$ como função de $\lambda$. Nestas figuras também se pode ver as correspondentes regiões ampliadas das vizinhanças do PMC.
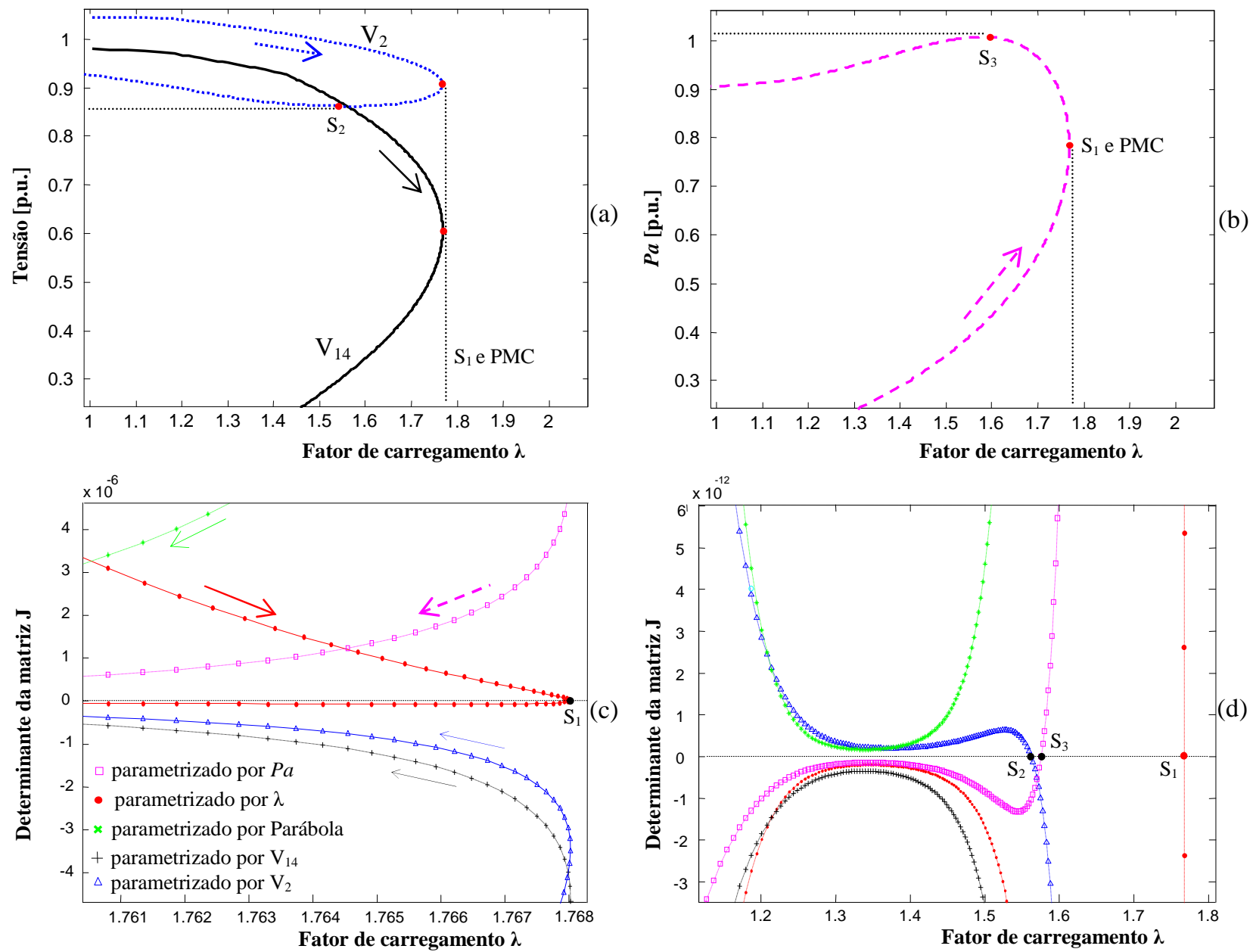

Figura 3: Pontos de singularidade para o sistema IEEE-14 barras: (a) curvas P-V, (b) curva $\lambda$-Pa, (c) determinante da matriz $\mathbf{J}$ para cada parâmetro, (d) região ampliada da figura (c) 
Observe nestes detalhes, que o encontro entre as duas curvas 1 e 2 se dá na parte de baixo da curva P-V, próximo ao PMC. Na Fig. 5 se apresentam para cada método, os correspondentes valores numéricos dos determinantes para os casos das curvas 1 e 2 . Observa-se que no caso da curva 1 o gráfico dos determinantes apresenta uma descontinuidade próximo ao PMC, mostrando que nesse caso o PMC é ocasionado por uma BIL, ou seja, uma bifurcação induzida por limites, no caso pelo limite de potência reativa do gerador 7062. Observe que se o seu valor for fixado em seu valor máximo desde o início do traçado da curva, o PMC seria ocasionado por uma BSN conforme se pode confirmar nas figuras.

Na Fig. 5(a) é apresentado as duas curvas de valores dos determinantes da matriz J (equação 2) do método parametrizado por $\lambda$. A curva 2 comprova que a singularidade do ponto $\mathrm{S}_{1}$ correspondente a uma BSN. Como se pode ver, no caso da curva 1 ocorreu uma descontinuidade na curva dos determinantes, a qual, conforme se constata nas regiões ampliadas das Figs. 4(a) e (b), acontece na porção correspondente a parte de baixo da curva P-V. Entretanto, da curva 1 apresentada na Fig. 4(a), se comprova que a singularidade do novo ponto $\mathrm{S}_{1}$ também é ocasionado por uma SNB e não por uma BIL.

Conforme apresentado nas Fig. 5(b), o método não apresenta problemas numéricos relacionados com a presença de singularidade se o parâmetro usado for magnitude de tensão da barra $526\left(\mathrm{~V}_{526}\right)$, equação 3 . Já no caso da parametrização por $\mathrm{V}_{7062}$, a singularidade ocorre apenas no ponto $\mathrm{S}_{2}$, ver o detalhe da Fig. 5 (c). Na Fig. 6 são apresentados as curvas dos valores numéricos dos determinantes das matrizes Jacobianas dos métodos parametrizados por parábolas (equação 5) e por $\mathrm{Pa}$ (equação 4). Observe na Fig. 6(a) que no caso da utilização das equações das parábolas não há singularidade da matriz Jacobiana em nenhuma das curvas. No entanto quando da parametrização por $\mathrm{Pa}$, a singularidade ocorre no ponto $\mathrm{S}_{3}$ da curva 2, Fig. 6(b). Observa-se na curva 1 da Fig. 6(b) que o gráfico do determinante da matriz Jacobiana apresenta um salto exatamente no ponto $S_{3}$, no entanto pode se constatar que o método utilizando Pa também identifica este ponto como sendo ocasionado por uma SNB, Fig. 6(c). Observe na fig 6(c) que o fator de carregamento em $\mathrm{S}_{1}$ é ligeiramente maior que o de $\mathrm{S}_{3}$.
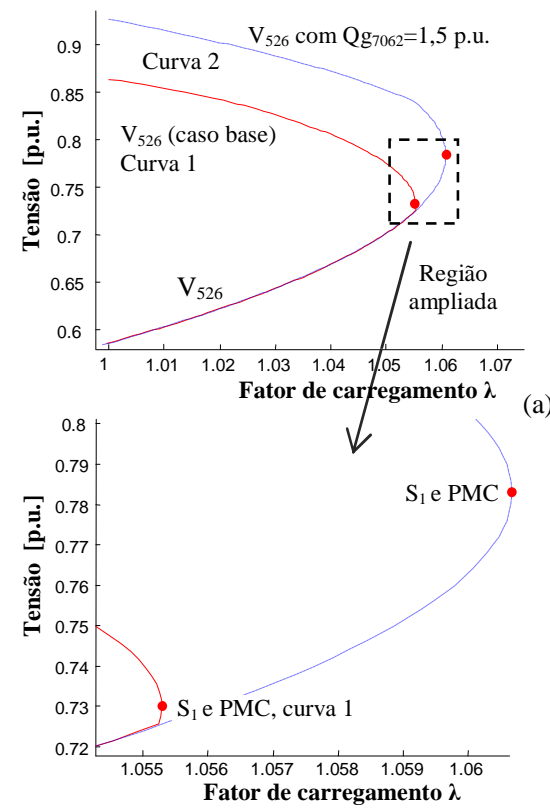

(a)

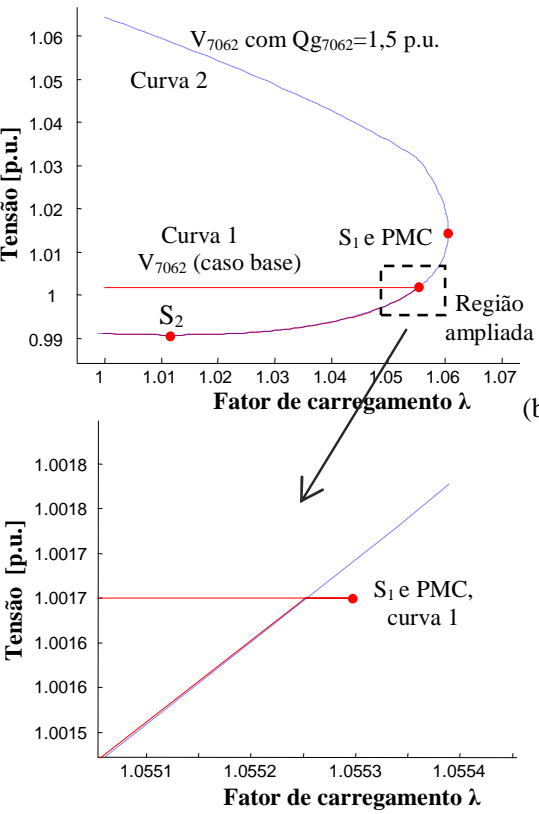

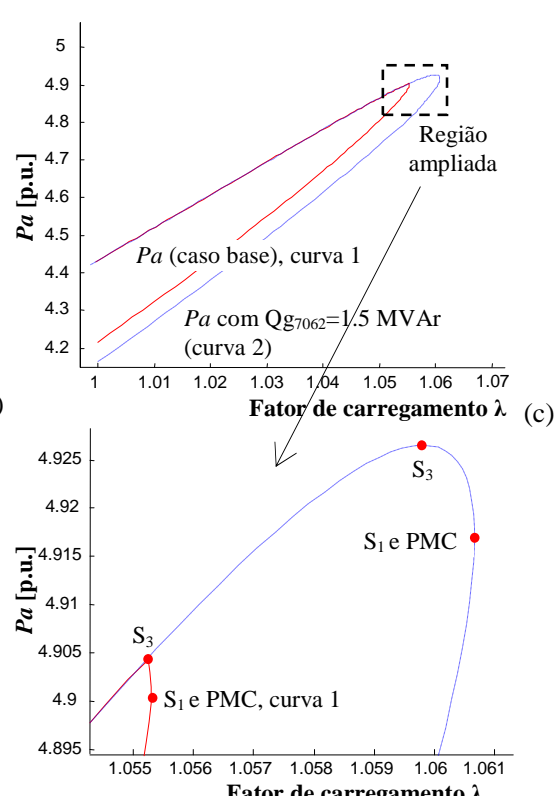

Fator de carregamento $\lambda$

Figura 4: Pontos de singularidade para o sistema IEEE-300 barras: (a) curvas P-V $V_{526}$ para o caso base e P-V $V_{526}$ para $Q_{7062}=1,5$ p.u. MVAr, (b) curvas $P-V_{7062}$, (c) curva $\lambda-P a$.
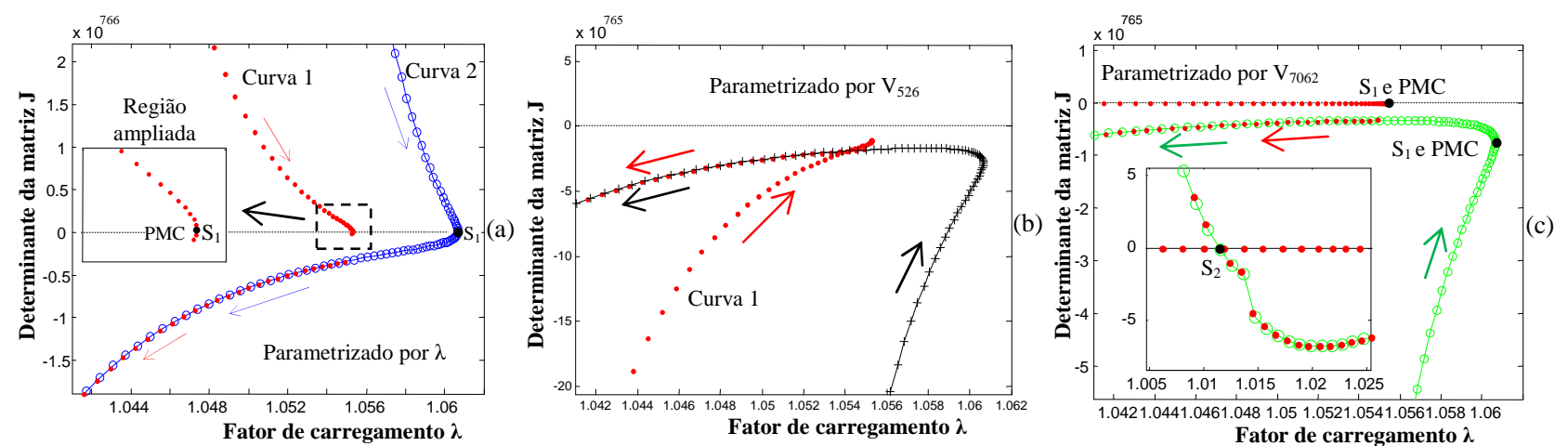

Figura 5: Determinante da matriz J: (a) parametrizado por $\lambda$, (b) parametrizado por $\mathrm{V}_{526}$, (c) parametrizado por $\mathrm{V}_{7062}$. 


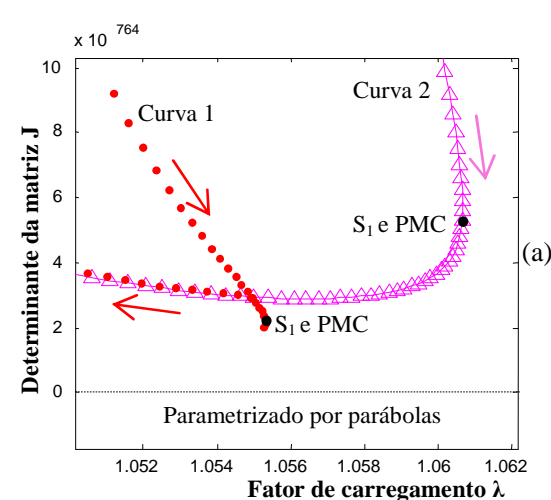

Figura 6: Determinante da matriz
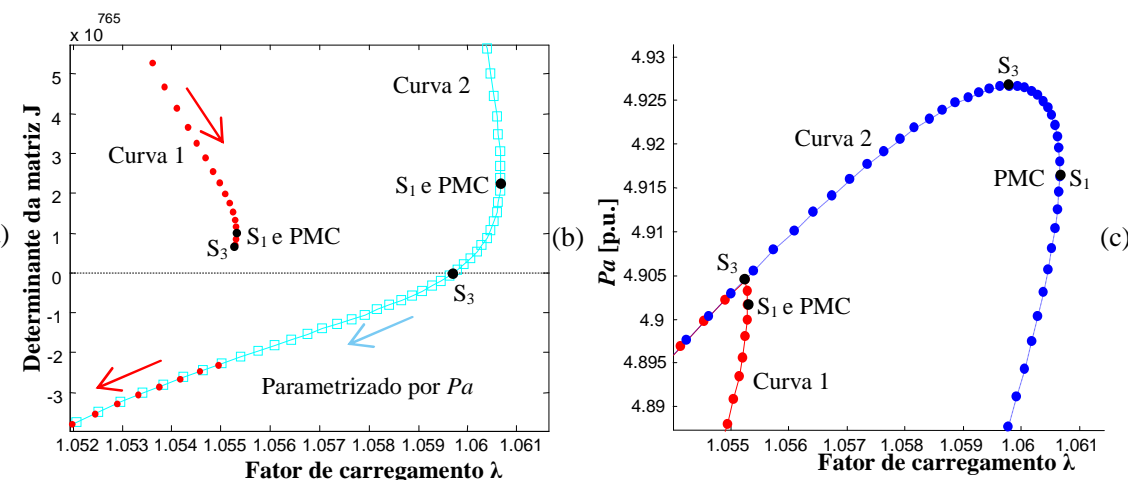

(c)

\section{CONCLUSÃO}

Neste trabalho apresentou um estudo de singularidade da matriz Jacobiana do fluxo de carga continuado. Foram apresentadas várias técnicas de parametrização geométrica para a obtenção do PMC de Sistemas Elétricos de Potência. Os parâmetros utilizados nas técnicas são: o fator de carregamento $(\lambda)$, a magnitude de tensão da barra crítica ou de uma barra específica, a equação das perdas totais de potência ativa $(P a)$, e a equação quadrática localizada no plano $\lambda-P a$, ou seja, parábolas.

O ponto de máximo carregamento pode ser consequente ou de uma bifurcação de sela-nó (BSN) relacionada ao limite físico de capacidade de transmissão num sistema elétrico, onde a matriz Jacobiana é singular, ou de uma bifurcação estática induzida por limites (BIL), relacionada com o limite de potência reativa do gerador, onde a matriz não é singular. Neste sentido, apresentou neste trabalho propostas para a melhoria do desempenho do fluxo de carga continuado visando à obtenção do ponto de máximo carregamento de um sistema elétrico de potência sem problemas numéricos relacionados à singularidade da matriz Jacobiana. Um estudo baseado no determinante da matriz Jacobiana foi efetuado com o intuito de verificar se o ponto de máximo carregamento obtido corresponde a uma bifurcação selanó ou a uma bifurcação estática induzida por limites.

\section{AGRADECIMENTOS}

Os autores agradecem o apoio do CNPq e da Fundunesp.

\section{REFERÊNCIAS}

WSCC-Reactive Power Reserve Work Group (RRWG), 1998. "Final Report: Voltage Stability Criteria, Undervoltage Load Shedding Strategy, and Reactive Power Reserve Monitoring Methodology", 145 p.

FTCT-Força Tarefa Colapso de Tensão, 1999. Critérios e Metodologias Estabelecidos no âmbito da Força - Tarefa Colapso de Tensão do GTAD/SCEL/GCOI para Estudos de Estabilidade de Tensão nos Sistemas Interligados Norte/Nordeste, Sul/Sudeste e Norte/Sul Brasileiros, XV SNPTEE, GAT-10, Foz do Iguaçu, PR,

Cañizares, C. A.; Alvarado, F. L.; DeMarco, C. L.; Dobson, I.; Long, W. F. 1992. Point of Collapse Methods Applied to AC/DC Power Systems. IEEE Trans. on Power Systems, v. 7, n. 2, p.673-683.

Alves D. A.; Silva, L. C. P.; Castro C. A.; Costa V. F. 2000. Continuation load flow method parameterized by power losses. IEEE Power Engineering Society Winter Meeting Conf., p.1123-1128.

Ajjarapu, V.; Christy, C. 1992. The Continuation Power Flow: a Tool for Steady State Voltage Stability Analysis. IEEE Trans. on Power Systems, v.7, n.1, p. 416-423.

Chiang, H. D.; Flueck, A.; Shah, K.S.; Balu, N. 1995. CPFLOW: A Practical Tool for Tracing Power System Steady State Stationary Behavior Due to Load and Generation Variations. IEEE Trans. on Power Systems, v.10, n.2, p. 623-634.

Yang X, Zhou X, Ma Y, Du Z. 2012. Asymptotic numerical method for continuation power flow. Electr Power Energy Syst., 43:670-9.

Pama A, Radman G. 2009. A new approach for estimating voltage collapse point based on quadratic approximation of PV-curves. Electr Power Syst Res., 79:653-9.

Li SH, Chiang HD. 2008. Nonlinear predictors and hybrid corrector for fast continuation power flow. IET Gener, Transm Distrib., 2(3):341-54.

Bonini Neto A, Alves DA. 2010. Improved geometric parameterization techniques for continuation power flow. IET Gener, Transm Distrib., 4(12):1349-59.

Magalhães, E. M. ;Bonini Neto, A.; Alves, D. A. 2013. Técnica de Parametrização Geométrica para o Fluxo de Carga Continuado Baseado nas Variáveis Fator de Carregamento e Perda Total de Potência Ativa. In: Décimo Quinto Encontro Regional Ibero-americano do CIGRÉ - XV ERIAC, Foz do Iguaçu, p. 1-8. 
Congresso Nacional de Matemática Aplicada à Indústria, 18 a 21 de novembro de 2014, Caldas Novas - GO

\section{RESPONSABILIDADE AUTORAL}

"Os autores são os únicos responsáveis pelo conteúdo deste trabalho". 


\title{
SINGULARITY STUDIES IN CONTINUATION POWER FLOW
}

\author{
Alfredo Bonini Neto ${ }^{1}$, e-mail: bonini@tupa.unesp.br \\ Dilson Amancio Alves², e-mail: dalves@dee.feis.unesp.br \\ ${ }^{1}$ UNESP - São Paulo State University - Campus Tupã, e-mail: bonini@tupa.unesp.br \\ ${ }^{2}$ UNESP - São Paulo State University - Campus Ilha Solteira, e-mail: dalves@ dee.feis.unesp.br
}

\begin{abstract}
In recent years the preoccupation with the voltage stability subject has been gaining attention worldwide when it refers to the energy sector industry, this is due to the fact that this issue is related to the operation and planning of electrical power systems. Factors such as the increasing demand for energy, the transfer of large amounts of power to meet the consumption, combined economic and environmental demands have led the systems operating under stressful conditions (near its limits), i.e., with small margins security that is a threat to their condition of stable operation. The combination of these factors can be disastrous, because become the electrical power systems vulnerable, or exposed to the risk of instability. In the literature, one of the studies for stability and voltage instability is related to $P-V$ curve (power versus voltage magnitude) and the point on the curve that separates stable of the unstable operation is called as maximum loading point. The maximum loading point can be consequent of a saddlenode bifurcation $(S N B)$ related to the limit of transmission capacity in a power system, where the Jacobian matrix is singular or limit induced bifurcation (LIB), related the limit of reactive power of the generator, where the matrix is not singular. In this sense, it is presented in this paper a proposal to improve the performance of the continuation power flow in order to obtain the maximum loading point of an electrical power system without numerical problems related to the Jacobian matrix singularity. A study on maximum loading point is made in order to check whether the point corresponds to a saddle node bifurcation (SNB) or a limit induced bifurcation (LIB).
\end{abstract}

Keywords: Voltage stability, Maximum load point, Bifurcation, Continuation power flow. 